\title{
Kooperation und Wettbewerb in Organisationen
}

\author{
Ralf Lanwehr ${ }^{1}$ Falko von Ameln ${ }^{2}$
}

Online publiziert: 20. Februar 2017

(C) Springer Fachmedien Wiesbaden 2017

\section{Thementeil ,Kooperation und Wettbewerb in Organisationen"}

In der betriebswirtschaftlichen wie in der psychologischen Forschung gewinnt seit Jahren eine Perspektive an Bedeutung, welche als Ablösung kontingenztheoretischer Überlegungen aufgefasst werden kann.

Während sich klassische Vertreter/-innen situativer Ansätze über die Frage Gedanken machen, unter welchen Bedingungen welche Form des Handelns sinnvoll sei (Woodward 1970; Lawrence und Lorsch 1967; Fiedler 1967), steht zunehmend die zeitgleiche Behandlung direkt konfligierender Themen im Vordergrund (Lado et al. 2006). Man könnte die damit verbundene Theorieschule vielleicht am besten als Dilemmatheorie bezeichnen (Lanwehr 2008). Konstitutiv für den Ansatz ist das Vorhandensein mindestens zweier sich scheinbar ausschließender Handlungsoptionen. Dies gilt für viele Phänomene mit Bezug zu den drei Ebenen Gruppe, Interaktion und Organisation, welche diese Zeitschrift schwerpunktmäßig diskutiert. Schließlich sollen Organisationen langfristige Strategien verfolgen, aber gleichzeitig gute Quartalszahlen vorweisen und sie sollen zwar das Produktportfolio diversifizieren, sich parallel aber auf ihr Kerngeschäft konzentrieren. Führungskräfte in Organisationen sollen Zeit- und Kostenvorgaben bindend einhalten, aber zugleich innovative Konzepte einbringen. Sie sol-

Ralf Lanwehr

lanwehr.ralf@fh-swf.de

1 Fachbereich Ingenieur- und Wirtschaftswissenschaften, Fachhochschule Südwestfalen, Lindenstr. 53, 59872 Meschede, Deutschland

2 FH Südwestfalen, Am Diekschloot 11b, 26506 Norden, Deutschland len den Arbeitsfortschritt der Geführten kontrollieren, dabei jedoch deren Wahl der Wege freilassen, um die Entwicklung der Geführten zu fördern (Lanwehr et al. 2013).

In der Theoriebildung wurde diesem Umstand bereits in verschiedener Hinsicht Rechnung getragen. So klassifizieren Smith und Lewis (2011) beispielsweise auf hoher Abstraktionsebene eine Taxonomie dilemmatheoretischer Theorieansätze. Raisch et al. (2009) würdigen in einer integrativen Betrachtung den Bereich der organisationalen Beidhändigkeit, Armbrüster und Gebert (2002) diskutieren dilemmatheoretische Phänomene der Organisationskultur, Simons (2005) beschäftigt sich mit Auswirkungen auf das Controlling, Sheremata (2000) modelliert dilemmatheoretisch das Innovationsmanagement, Lawrence et al. (2009) beziehen sich auf Führung und Smith und Tushman (2005) auf die Geschäftsführung.

Ein Beispiel für ein solches Spannungsfeld ist das Verhältnis von Kooperation und Wettbewerb in Organisationen.

Das im Zusammenhang mit dem strategischen Management diskutierte Konzept der Coopetition beschreibt das Phänomen einer interorganisationalen Beziehung, welche von Kooperation und Wettbewerb zugleich geprägt ist und sich deshalb auch aus den Begriffen Cooperation und Competition zusammensetzt (Brandenburger und Nalebuff 1996). Allerdings existiert dieses Konzept parallel und relativ abgekoppelt von den zuvor dargestellten Überlegungen - in einer Synthese des aktuellen Sachstandes der Diskussion finden sich keinerlei Überschneidungen zu den genannten dilemmatheoretischen Ansätzen (Bouncken et al. 2015). Hinzu kommt für das Phänomen des zeitgleichen Vorliegens von Kooperation und Wettbewerb der Umstand, dass bislang vor allem Beziehungen auf organisationaler Ebene thematisiert wurden (Bengtsson et al. 2016).

Für Interaktionen auf anderen Aggregatniveaus finden sich hingegen bislang nur wenige Ansätze. So weisen Luo 
et al. (2006) etwa einen Zusammenhang zwischen Coopetition innerhalb von Firmen nach, Bogers et al. (2017) thematisieren den Nutzen von Coopetition im Rahmen der Open-Innovation-Bewegung, Zhang et al. (2010) widmen sich der interkulturellen Spezifika, Bullinger et al. (2010) untersuchen politische Gemeinden und Pareto et al. (2012) Lerneffekte unter den gleichzeitigen Bedingungen von Kooperation und Wettbewerb.

Was jedoch weitgehend fehlt, ist eine anwendungsorientierte Betrachtung des Spannungsverhältnisses aus Kooperation und Wettbewerb innerhalb von Organisationen und ihrer untergeordneten Ebenen. Welche konkreten Ansätze existieren und wie erfolgsbedeutsam sind sie?

Diesem Thema, nämlich der anwendungsorientierten Betrachtung von Kooperation und Wettbewerb, widmet sich die vorliegende Ausgabe.

Eveline Mäthner und Ralf Lanwehr führen in das Thema ein, indem sie an Grant anknüpfen, der drei Stile von intraorganisationalem Austauschverhalten vorstellt: „Geber“, „Nehmer“ und „Tauscher“. Dieses Modell wird mit der Diskussion um prosoziales Verhalten und Organizational Citizenship Behavior (OCB) in Beziehung gesetzt. Die Autor/-innen plädieren dafür, motivationale Faktoren stärker $\mathrm{zu}$ berücksichtigen und unterscheiden Eigeninteresse (selfinterest), Aufopferung (self-sacrifice) und Orientierung an überindividuellen Interessen (collective rationality). Eine solche differenzierte Sicht, so die Autor/-innen, kann dazu beitragen, mögliche Nebeneffekte von OCB wie Stress oder Leistungsmängel besser zu verstehen. Der Artikel endet mit einem Ausblick auf die Implikationen der diskutierten Konzepte für Teams und Organisationskultur.

Kurt Smits Beitrag setzt sich auf der Grundlage spieltheoretischer Überlegungen mit dem Nutzen von Kooperation für Organisationen auseinander. Smits Ziel besteht insbesondere darin, die Überlegenheit von Kooperationskulturen gegenüber Wettbewerbskulturen zu belegen. Seine Argumentation fußt nicht nur auf der Spieltheorie, sondern bezieht auch Erkenntnisse der Neurobiologie ein. In seinem sehr praxisbezogenen Artikel leitet Smit Empfehlungen für die Gestaltung von Führung und Fehlerkultur ab.

Henning Staar und Hans Christian Atzpodien befassen sich in ihrem Beitrag mit der Fragestellung, inwieweit überfachliche personale Kompetenzen (Einflusskompetenz, Netzwerkkompetenz) für die Arbeit in Netzwerken förderlich sind. Diese Fragestellung wird in einem Netzwerk von Ärzten anhand der Aussagen von 37 Probanden untersucht. Die Forschung zu Netzwerken ist ein wichtiger und ausgesprochen aktueller Zweig der Organisationspsychologie, $\mathrm{zu}$ dem bislang noch zu wenig Forschung existiert. Insofern ist es erfreulich, dass der vorliegende Artikel einen konkreten und nicht nur wissenschaftlich, sondern auch praktisch hochrelevanten Aspekt in den Blick nimmt und empirisch beforscht. Auf der Basis ihrer Studie kommen die
Autoren zu der Empfehlung, bei Aufbau und Gestaltung von Gesundheitsnetzwerken neben strategischen Fragen in stärkerem Maße personenbezogene Kompetenzen zu entwickeln und zu fördern.

Sabine Boerner, Hendrik Hüttermann und Max Reinwald analysieren den Stand der Forschung zur Rolle von Führung in heterogen zusammengesetzten Teams aus theoretischer und empirischer Perspektive. Sie zeigen, dass Führung in heterogenen Teams dann besonders effektiv ist, wenn sie auf die Diversity-Merkmale der Teammitglieder abstimmt ist. Aus dieser Erkenntnis werden Empfehlungen zur Führung heterogener Teams und zur Förderung diversitybezogener Führungskompetenzen in Unternehmen abgeleitet.

\section{Offener Teil}

Auch der erste Beitrag im themenungebundenen Teil der Zeitschrift befasst sich mit dem Thema Kooperation: Dölf Looser untersucht in einer quasi-experimentellen Studie, wie sich eine erlebnispädagogische Projektwoche auf Kooperation vs. Ausgrenzung in einer Schulklasse auswirkt.

Julia Seelandt, Bastian Grande und Michaela Kolbe vom Universitätsspital Zürich untersuchen, wie Führungskräfte im Krankenhaus (Anästhesisten/-innen und Pflegefachpersonen) ihre Rolle in medizinischen Akutsituationen auffassen und welche Auswirkungen diese impliziten Führungstheorien auf die Zusammenarbeit haben.

Der dritte Beitrag im offenen Teil greift ein ebenso wichtiges wie aktuelles Thema auf, das wir in Heft 3/2016 der GIO intensiv beleuchtet haben: das Thema Flucht und Migration in seinen Implikationen für Organisationen. Julia A. M. Reif, Erika Spieß und Rita Berger entwickeln ein Modell, das Herausforderungen und Chancen bei der Integration von Arbeitsmigrant/-innen in Organisationen aufzeigt.

\section{Aktuelles: Achtsamkeit}

In unserer Rubrik „Aktuelles“ nehmen wir aktuelle Trends und Diskurse in Theorie und Praxis der Organisationspsychologie in den Blick. Im vorliegenden Heft setzt sich Telse Iwers mit dem Konzept der Achtsamkeit auseinander und beschreibt einen eigenen Ansatz für eine achtsamkeitsbasierte Praxis.

\section{Call for Papers}

Unser Anspruch ist es, zukunftsweisende Erkenntnisse im Spannungsfeld von Gruppe, Interaktion und Organisation zu präsentieren. Der Komplexität des Gegenstandsbereichs entsprechend beziehen wir - ausgehend von einer Verortung 
in der Organisationspsychologie - relevante Erkenntnisse aus den Nachbardisziplinen (z. B. Organisationssoziologie, BWL, Philosophie) ein. Unser Anspruch besteht dabei darin, als Vermittlerin zwischen Theorie und Praxis zu wirken, d. h. Beiträge sollen für die scientific community ebenso anschlussfähig sein wie für Berater/-innen, Supervisor/-innen, Coaches und Führungskräfte.

Die Redaktion freut sich über exzellente Beiträge, die dieser Zielsetzung entsprechen. Einreichungen sind möglich für den offenen Teil sowie für die Schwerpunktthemen der kommenden Hefte:

- Organisationsdynamik Heft 3/17 (bis 01.04.2017),

- Führung in Zeiten der Digitalisierung Heft 4/17 (bis 31.07.2017),

- Karriere Heft 1/18,

- Achtsamkeit Heft 2/18.

Nähere Informationen, Autorenhinweise etc. finden Sie auf www.springer.com/11612.

Allen Leserinnen und Lesern wünschen wir eine erkenntnisreiche Lektüre.

\section{Ralf Lanwehr, Falko von Ameln}

\section{Literatur}

Ambrüster, T., \& Gebert, D. (2002). Uncharted territories of organizational research: the case of Karl Popper's Open Society and its enemies. Organization Studies, 23(2), 169-188.

Bengtsson, M., Kock, S., Lundgren-Henriksson, E.-L., \& Näsholm, M.H. (2016). Coopetition research in theory and practice: growing new theoretical, empirical, and methodological domains. Industrial Marketing Management, 57(1), 4-11.

Bogers, M., Zobel, A.-K., Afuah, A., Dahlander, L., Gruber, M., Hilgers, D., Majchrzak, A., et al. (2017). The open innovation research landscape: established perspectives and emerging themes across different levels of analysis. Industry and Innovation, 24(1), $8-40$.

Bouncken, R. B., Gast, J., Kraus, S., \& Bogers, M. (2015). Coopetition: a systematic review, synthesis, and future research directions. Review of Managerial Science, 9(3), 577-601.

Brandenburger, A.M., \& Nalebuff, B.J. (1996). Co-opetition. New York: Doubleday.

Bullinger, A.C., Neyer, A.-K., Rass, M., \& Möslein, K. (2010). Community-based innovation contests: Where competition meets cooperation. Creativity \&amp; Innovation Management, 19(3), 290-303.

Fiedler, F.E. (1967). A theory of leadership effectiveness. New York: McGraw-Hill.

Lado, A. A., Boyd, N. G., Wright, W., \& Kroll, K. (2006). Paradox and theorizing within the resource-based view. Academy of Management Review, 31(1), 115-131.

Lanwehr, R. (2008). Zum Zusammenhang von Führungsverhalten und Führungserfolg - Eine Diskussion aus dilemmatheoretischer Perspektive. Berlin: TU Berlin.

Lanwehr, R., Müller-Lindenberg, M., \& Mai, D. (2013). Balance Management - Vom erfolgreichen Umgang mit gegensätzlichen Zielen. Heidelberg: Springer.
Lawrence, P., \& Lorsch, J. W. (1967). Differenciation and integration in complex organizations. Administrative Science Quarterly, 12(1), $1-47$.

Lawrence, K. A., Lenk, P., \& Quinn, R.E. (2009). Behavioral complexity in leadership: the psychometric properties of a new instrument to measure behavioral repertoire. Leadership Quarterly, $20(2), 87-102$.

Luo, X., Slotegraaf, R. J., \& Pan, X. (2006). Cross-functional "Coopetition": the simultaneous role of cooperation and competition within firms. Journal of Marketing, 70(2), 67-80.

Pareto, L., Haake, M., Lindström, P., Sjödén, B., \& Gulz, A. (2012). A teachable-agent-based game affording collaboration and competition: evaluating math comprehension and motivation. Education Technology Research Development, 60(5), 723-751.

Raisch, S., Birkinshaw, J., Probst, G., \& Tushman, M.L. (2009). Organizational ambidexterity: balancing exploitation and exploration for sustained performance. Organization Science, 20(4), 685-695.

Sheremata, W. A. (2000). Centrifugal and centripetal forces in radical new product development under time pressure. Academy of Management Review, 25(2), 398-408.

Simons, R. (2005). Levers of organization design: How managers use accountability systems for greater performance and commitment. Boston: Harvard Business School Press.

Smith, W. K., \& Lewis, M.W. (2011). Toward a theory of paradox: a dynamix equilibrium model of organizing. Academy of Management Review, 36(2), 381-403.

Smith, W. K., \& Tushman, M.L. (2005). Managing strategic contradictions: a top management model for managing innovation streams. Organization Science, 16(3), 522-536.

Woodward, J. (1970). Industrial irganisation: behaviour and control. Oxford: Oxford University Press.

Zhang, H., Shu, Z., Jiang, X., \& Malter, A. J. (2010). Managing knowledge for innovation: the role of cooperation, competition, and alliance nationality. Journal of International Marketing, 18(4), 74-94.

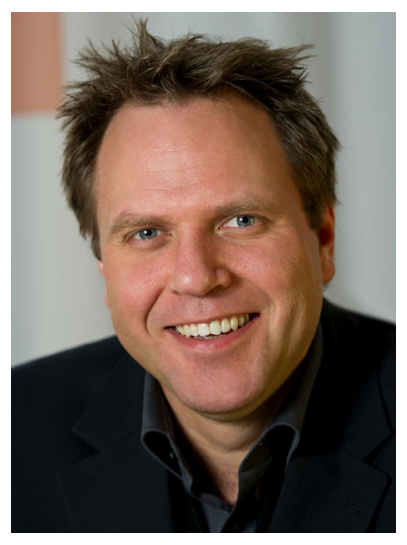

Ralf Lanwehr ist Professor für Wirtschaftspsychologie und hat sich auf evidenzbasiertes Management spezialisiert. Er steuert aktuell Drittmittelprojekte mit einer Förderhöhe von rund $€ 3$ Mio in enger Partnerschaft mit diversen DAX30Firmen, darunter auch BMW. Als Geschäftsführer des Netzwerks Fußballanalytik hat er zudem sein Hobby zum Beruf gemacht und ist für verschiedene Vereine der ersten Bundesliga tätig. Die große Karriere als Profifußballer blieb nämlich trotz seiner Zeit als Stürmer von Balane Inhambane in der dritten mosambikanischen Liga verdientermaßen aus. 


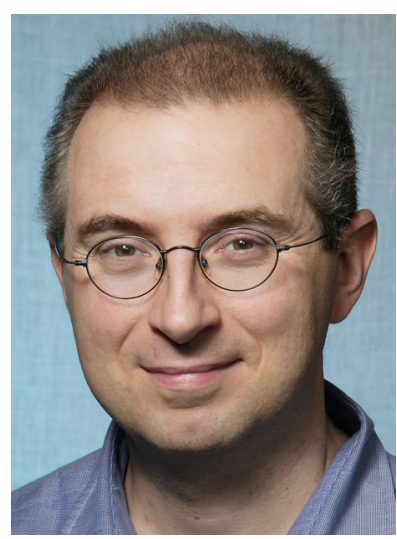

Falko von Ameln Priv.-Doz. Dr. phil. Dipl.-Psych., Organisationsberater mit Arbeitsschwerpunkten Change Management, Führungskräfteentwicklung und Aus-/ Fortbildung von Berater/innen, Supervisor/innen und Coaches. Habilitation im Fach Beratungswissenschaft. Zahlreiche Publikationen zu Theorie und Methodik der Beratung sowie Führung. 